\title{
À la Rencontre de Deux Antinationalistes: La Pensée Nationaliste de Trudeau et Kedourie
}

Maxime Perreault

l'Université de Laval

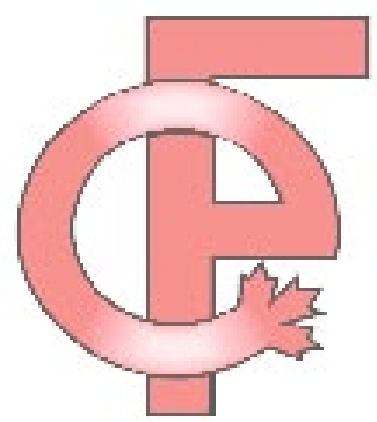

Maxime Perreault est étudiant en science politique à I'Université Laval où il termine actuellement son baccalauréat. À l'automne 2006, il entreprendra une maîtrise dans ce Même domaine à cette université. II travaille actuellement comme auxiliaire de recherche au Centre d'analyse des politiques publiques de I'Université Laval. Ses intérêts sont les politiques publiques (particulièrement en santé) et la sociologie politique 


\section{Introduction}

Dans son livre La voie canadienne : repenser le fédéralisme, Will Kymlicka soutient deux modèles de fédéralisme existent tout dépendamment de la source de diversité qu'il vise à concilier avec l'unité (objectif du fédéralisme). Le premier modèle est le modèle territorial où la diversité géographique, démographique ou économique entre les territoires s'institutionnalise pour créer une fédération divisée (en pouvoir et en territoire) sur cette différence. Le second modèle est le modèle ethnique, culturel ou national. Dans ce modèle, c'est la présence de minorités nationales possédant des traditions, langues ou institutions distinctes de la majorité qui provoque une diversité. Le fédéralisme basé sur cette différence distribuera donc des pouvoirs ou des territoires en fonction de la présence des nations. ${ }^{i}$

Par contre, l'association entre nation(s) et État est souvent mal perçue dans un certain milieu politique. En effet, certains auteurs dénoncent tout nationalisme, qui divisent et attisent les différences, sous prétexte qu'il ne peut se concilier avec les objectifs d'universalité et de fraternité de la modernité politique. "Le courant antinationalisme était né et, de par son rejet de tout nationalisme, il a eu un impact considérable sur la pensée fédéraliste (privilégiant de façon notoire un fédéralisme territorial) et notamment au Canada.

Pour mieux comprendre cette situation qui a eu des impacts retentissants, il importe de bien saisir la pensée antinationaliste. Pour ce faire, nous allons procéder en deux temps. Premièrement, nous allons décrire la pensée de la figure emblématique de l'antinationalisme, Elie Kedourie. Au cours de l'analyse, nous nous attarderons à sa compréhension de l'origine et des problèmes du nationalisme. Deuxièmement, nous allons décrire la pensée de Pierre Trudeau, nettement influencé par ce dernier. Au départ, sa pensée sera très antinationaliste, mais au fil des événements, sa pensée se rattachera à un nationalisme civique imbriqué dans la Charte canadienne des droits et libertés. Dans cette partie, nous nous attarderons donc à cette évolution afin de démontrer comment l'antinationalisme de départ a eu une importance sur la formulation d'un nationalisme civique.

\section{A) L'anti-nationalisme d'Elie Kedourie}

\section{Contexte du développement de sa pensée}

La compréhension d'un auteur est incomplète sans la connaissance de son histoire. D'ailleurs, les importants reproches que Kedourie dresse à l'endroit du nationalisme sont évidemment influencés par un contexte historique spécifique. L'association entre violence et nationalisme et entre nation et déstabilisation sont nourries par deux événements historiques. Dans le contexte de l'aprèsguerre, plusieurs auteurs ont associé le nationalisme à la violence hitlérienne. Kedourie en fait partie. iii En effet, en remontant aux bases philosophiques du nationalisme théorisées par Herder et Fichte, il dresse un lien entre l'idéologie nazie et ces derniers." Autrement dit, le nationalisme est forcément violent, la preuve étant la Shoah. On comprend donc le lien qui unit cet événement historique à la théorie de Kedourie. Ensuite, il faut noter que «le livre de Kedourie sur le nationalisme fut publié pour la première fois en 1960, alors que 
l'Europe et l'Afrique étaient empêtrées dans la difficile entreprise de la décolonisation. " "On peut facilement comprendre que la vague de décolonisation vient confirmer les appréhensions de Kedourie sur le nationalisme puisque ce "nationalisme décolonisateur" est généralement violent et déstabilisateur. La décolonisation vient donc rassurer l'analyse sévère que Kedourie dresse du lien entre nationalisme, violence et déstabilisation.

À présent que nous connaissons le contexte historique de son œeuvre, nous pouvons maintenant nous attarder à la théorie proprement dite.

\section{La nation : résultat idéologique et moderne!}

Tout d'abord, pour bien comprendre les théories nationalistes de Kedourie, il est important de comprendre comment ce dernier explique les causes historiques de l'avènement du nationalisme. Sur ce point, deux écoles s'affrontent. La première affirme que l'origine précède considérablement la modernité politique La séconde affirme plutôt que le nationalisme fest le résultat de cette modernité. Par exemple, Gellner affirme que les identités nationales sont nées avec l'industrialisation tandis qu'Anderson affirme qu'elles sont nées avec la chute de la monarchie et le développement de l'imprimerie. ${ }^{\text {vi }}$ Kedourie appartient à la seconde école. Par contre, ce dernier n'affirme pas, comme Gellner ou Anderson, que cette idéologie se développe à cause de causes socio-économiques. Il affirme que le nationalisme est le résultat de la philosophie d'Emmanuel Kant :
«Doctrine de l'autodétermination, le nationalisme a subi l'influence de la philosophie de Kant. Dans le système kantien, l'autonomie morale s'acquiert au prix d'un combat terrible. La doctrine est volontariste : ne sont rationnelles et légitimes que les limites que l'on s'impose à soi-même. Autour de cet extrémisme intransigeant et de ce volontarisme, le style nationaliste commence à prendre forme. Il se précisera davantage avec l'entrée en scène des penseurs $\mathrm{du}$ romantisme allemand, notamment Herder et Fichte, qui font suffoquer les individus sous l'étreinte du Léviathan étatique. Kedourie est catégorique dans ses interprétations : pour Fichte, c'est l'État qui est le créateur de la liberté de l'individu [...]. Pour Herder, l'épanouissement individuel ne se réalise que grâce à l'absorption dans l'État. "iii "

On voit donc que l'origine du nationalisme est idéologique et remonte à la réaction romantique face aux idéaux de Kant. ${ }^{\text {ix }}$ Or, le côté philosophique du nationalisme permet à Kedourie de conclure que le tout " est créé de toutes pièces par des élites et passivement endossés par des peuples inertes et facilement manipulables. " A Ainsi, on peut comprendre l'accent que met Kedourie sur les acteurs démagogues de même que les problèmes qu'il associe à l'utilisation de la doctrine nationaliste. Ces problèmes se divisent en deux: politiques et philosophiques.

\section{Problèmes politiques}

Dans la deuxième section de son livre (chapitre 6 et 7), Kedourie présente une quantité incroyable de 
problèmes politiques reliés au nationalisme. On peut les regrouper sous deux sections : la violence et la déstabilisation. Tout d'abord, Kedourie affirme que la naissance du nationalisme s'est produite à une époque violente. ${ }^{\mathrm{xi}}$ Il affirme que, puisque cette doctrine est née dans la violence, elle appelle forcément à la violence. Il démontre le tout en expliquant que certains acteurs nationalistes appelaient au coup d'État, à l'insurrection et à la guerre civile. ${ }^{\text {xi }}$ Aussi, en décrivant certains événements du XIXe et XXe siècles, il affirme que «des crimes parmi les plus honteux ont été indiscutablement commis au nom du nationalisme. " xiii Donc, en regardant la naissance de l'idéologie, les discours des différents acteurs nationalistes et des événements historiques, Kedourie conclut que nationalisme va de pair avec la violence. Également, il affirme que le nationalisme débouche forcément à la violence à cause de son refus du compromis : "The settlement must be final. There can be no compromise. No halfway decision would be tolerable ${ }^{\text {iv }}$ " Or, la sociologie politique nous apprend que, lorsque le compromis est impossible, il ne reste que la violence (ou l'abnégation) pour arriver à ces fins. Finalement, Kedourie croit que le nationalisme vise le rassemblement sous un toit politique (État et territoire) d'une communauté linguistique. Étant donné qu'une langue est rarement concentrée sur un même territoire, il craint qu'une guerre soit faite pour rassembler les gens d'une même langue sur un même territoire. Par exemple, si le Québec repose sur des frontières linguistiques, il devrait posséder une partie du Nord de l'Ontario et du Nouveau-Brunswick. Donc, une nation, par sa volonté d'unifier territoire et langue, conduira nécessairement à la violence ! Par contre, Kedourie n’a pas terminé de nous peindre un tableau sombre du nationalisme : il le noircit davantage en analysant le potentiel déstabilisateur.

Ensuite, le second problème que Kedourie associe au nationalisme est son effet déstabilisateur. L'argumentation est découpée en trois parties. Premièrement, Kedourie affirme que le nationalisme risque de conduire au déséquilibre interne dans la population. Pour prouver le tout, il réfère à cette constatation : les principaux militants des corpuscules nationalistes sont des jeunes et des gens de la classe moyenne. ${ }^{\mathrm{x}}$ Il termine donc le raisonnement en affirmant que l'institutionnalisation du nationalisme risque donc de provoquer des conflits de générations et des conflits de classes, puisqu'il ne fait pas l'unanimité dans la société. Deuxièmement, le nationalisme risque de déstabiliser l'équilibre dans les relations internationales. La logique qui sous-tend le tout est le suivant: à partir de sa compréhension de l'origine philosophique du nationalisme, il affirme que cette idéologie vise la reconnaissance de la diversité des nations et que « si ces dernières veulent à tout prix leur intégrité, elles souhaiteront nécessairement se transformer en États. ${ }^{\text {xi }}$ "Donc, il croit que le nationalisme débouche absolument vers la création d'un État-Nation et nous savons que, plus il y a d'État dans le système international, plus ce dernier est difficile à gérer. Donc, à ses yeux, l'affirmation nationale déstabilise les relations internationales. Troisièmement, Kedourie affirme que le nationalisme ne fait pas heureux ménage avec 
un État plurinational. Sur ce point, Guy Laforest commente :

"Une des thèses principales de Kedourie veut que le nationalisme ne fasse qu'accentuer les difficultés inhérentes à la vie politique dans les États pluriethniques, multinationaux. Les revendications nationalistes dans de telles sociétés ne feront qu'exacerber les tensions, elles feront vaciller les équilibres fragiles réagissant les rapports entre les différents groupes. En lieu et place de la stabilité et de la liberté, le nationalisme ne mène qu'à la haine mutuelle. ${ }^{\text {xii }}$ "

Kedourie croit que le nationalisme se fait contre un autre groupe. À partir de ce raisonnement et sachant qu'il associe nationalisme à la violence, il est normal de croire à la déstabilisation inhérente des États multiculturels. L'exemple que Kedourie donne pour confirmer le tout est les millets de l'Empire Ottoman. Pendânt près de cinq siècles, la tolérance a été observée à caùse des millets (droits politiques particuliers accordés aux communautés en fonction des différentes religions). Par contre, "the millet system broke down because such limited autonomy could not satisfy the nationalist's ambitions. ${ }^{\text {xiii } " ~}$ Donc, par sa volonté d'avoir plus ou les mêmes droits et libertés que les autres, le nationalisme a conduit à l'intolérance et à la chute des millets. À partir de ce raisonnement, il suggère aux dirigeants d'États de ne pas se résoudre à donner de l'importance au nationalisme (par exemple, par l'autonomie culturelle), parce que l'empirisme démontre que la situation apporterait la déstabilisation. ${ }^{\text {xix }}$ On voit donc poindre qu'au-delà des problèmes politiques, il y a aussi des problèmes philosophiques (par exemple, la tolérance) reliés au nationalisme.

\section{Problèmes philosophiques}

Dans son livre Nationalism, Kedourie ne se contente pas seulement d'identifier des problèmes politiques au nationalisme. En effet, dans la première partie où il dresse la genèse du nationalisme, il identifie six problèmes philosophiques. Premièrement, le nationalisme, en se basant sur les passions, les émotions et l'affectivité, évacue la Raison. En plus de favoriser les sophistes et les démagogues, Kedourie croit que l'individu perd sa valeur. Tout comme Kant, il affirme que la valeur la plus importante est l'autonomie ou le selfdetermination : "A good man is an autonomous man, and for him to realize his autonomy, he must be free. Self-determination thus becomes the supreme political good. ${ }^{x x}$ ". Ainsi, l'individu doit se donner lui-même des principes moraux à l'aide de sa Raison (et non de l'affectivité de la religion par exemple), ce qui nécessite certaines libertés individuelles. Donc, un régime qui bafoue la Raison pour l'affectivité réduit considérablement la valeur (ou la dignité) et la liberté de l'individu. Deuxièmement, à partir de ce problème, Kedourie craint que le nationalisme évacue l'individu. Il affirme que les deux premiers penseurs du nationalisme, Herder et Fichte, ne faisait qu'une place mineure à l'individu: pour ces philosophes, «l'épanouissement individuel ne se réalise que grâce à l'absorption dans l'État", représentant de la 
nation. ${ }^{\text {xi }}$ Or, la nation ne représente pas tous les individus pour Kedourie. ${ }^{\text {xii }}$ Donc, l'État Nation risque de nier certaines individualités. Troisièmement, un autre problème réside dans le fait que le nationalisme tire son origine du romantisme. Tout comme la philosophie romantique, le nationalisme n'accepte pas la nature initiale de l'homme: elle désire la changer. Or, Kedourie associe le tout aux pires dérives totalitaires et aux pires génocides de l'histoire; le fait que certains artistes romantiques mettent un accent sur la mort lui sert de preuve à ces récusations. ${ }^{\text {xiii }}$ Quatrièmement, le nationalisme entraîne une confusion très importante entre le privé et le public. L'exemple que Kedourie donne est le suivant: "Love may be private, and in it would be included love of family and love of friend; or it may be public, and this love of fatherland, or of religion, etc. ${ }^{\text {xiv } " ~ E n ~ p l u s ~}$ d'imputer des sentiments ou des parcelles d'affectivité dans l'espace public, le nationalisme politise certains aspects de la vie privée. Ainsi, comme tous les libéraux, Kedourie craint la perte de la liberté individuelle. Cinquièmement, Kedourie croit que le nationalisme mène forcément à l'intolérance. En ranimant certaines différences, l'État Nation risque d'agir en fonction de la majorité nationale et de persécuter les minorités. Le cas des millets, présenté précédemment, hante l'esprit de Kedourie sur ce point. Sixièmement et dernièrement, Kedourie constate que le nationalisme présente une interprétation du passé pour susciter l'action présente. ${ }^{\mathrm{xv}}$ Il craint donc que cette référence au passé en vienne à évacuer le progrès, dicté par la Raison humaine. En résumé, Kedourie croit que le nationalisme ne présente que des inconvénients pour la modernité. Il croit aussi que nationalisme et libéralisme sont incompatibles. ${ }^{x x i}$ En bafouant la tolérance, la neutralité étatique, l'individualisme, le progrès, la doctrine libérale en est évincée. De plus, nous savons que la démocratie obéit à des fondements libéraux. Dans une époque marquée par le nazisme, le fascisme et le socialisme soviétique, on peut croire qu'il craint que le nationalisme se transforme en totalitarisme, comme l'Allemagne nazie et la décolonisation lui font craindre !

\section{Nationaliste ou Anti-nationaliste?}

À la lumière de toutes les considérations, nous pouvons synthétiser la théorie de Kedourie. Nous voyons que ce dernier condamne le nationalisme ethnique à cause de sa proximité avec le totalitarisme, le romantisme, la violence et la non-modernité. Par contre, il ferait faux de conclure que l'auteur condamne tous les nationalismes: un nationalisme est acceptable s'il se conjugue avec les idéaux libéraux modernes. En d'autres mots, il accepte un nationalisme "based on the will.. xxii " Cependant, on ne peut pas affirmer que Kedourie endosse tout nationalisme civique, puisqu'un nationalisme moderne doit respecter la Raison, la liberté, l'individualité, le progrès, la tolérance, la dichotomie privée publique et la nature de l'homme. Bien que la nation civique soit inclusive et respecte certains principes du libéralisme, Kedourie refuse que le nationalisme soit affectif, puisqu'il évacue la Raison. xxiii Par exemple, Kedourie refuserait le nationalisme de la Charte canadienne des droits et libertés, même s'il est civique, puisqu'il vise à construire une nation 
canadienne grâce à des sentiments (dans la seconde section, nous ferrons la preuve de ceci). Par contre, il est d'accord que l'État fasse la promotion du multiculturalisme, puisque cela va de pair avec l'idée de neutralité étatique, de tolérance, de liberté et d'individualisme.

Ainsi, aux yeux de Kedourie, le nationalisme canadien obtiendrait une très mauvaise note. Son insistance sur l'émotivité est nettement condamnable. ${ }^{\text {xix }}$ Cependant, pendant une très longue période, le « concepteur " de ce nationalisme, Pierre Trudeau, a été très près des idéaux anti-nationalistes de Kedourie. Par contre, le contexte politique canadien a eu raison de la philosophie de Trudeau : il s'est finalement résolu à utiliser le « ciment ». Cette situation signifie-t-elle que Kedourie a tort dans cette conception de la nation ? En présentant les deux phases de la pensée de Trudeau, nous espérons le découvrir.

B) Pierre Elliott Trudeau : pour et contre Kedourie?

Pierre Trudeau se présente à la fois comme défenseur et comme critique de l'œuvre de Kedourie. Dans la première phase de sa pensée, il met sur pied un système près de la vision du précédent auteur. Par contre, déjà en 1962, on note des divergences entre la pensée de Trudeau et celle de son «maître ". L'origine du nationalisme en témoigne.

\section{Les origines du nationalisme}

Au lieu d'associer le développement de la pensée nationaliste uniquement à l'idéologie comme chez Kedourie, il associe le nationalisme à une panoplie de causes philosophiques, sociologiques et politiques, comme le présente la citation suivante :

« Enfin, après plus de 65 siècles d'histoire, avec l'éclatement de l'ordre médiéval, la régression du latin comme langue de l'homme lettré, et la naissance de la mystique individualiste, la notion moderne de la nation commença à se développer. Le remplacement de l'Église catholique par des Églises nationales, la montée des bourgeoisies, le mercantilisme protecteur des économies territoriales, les outrages commis contre certains groupes ethniques [...], ce furent des facteurs qui contribuèrent à donner naissance aux aspirations nationales, celles-ci devant conduire à la mise sur pied successive des États nationaux. ${ }^{\mathrm{xxx}}$ "

On voit donc que Trudeau affirme la naissance de cette doctrine est liée à des facteurs religieux, linguistiques, politiques et économiques. Par contre, on ne peut pas conclure que ce personnage se situe dans l'école moderniste qui associe la naissance du nationalisme à la modernité. À première vue, on peut même affirmer l'inverse. En effet, Trudeau affirme que le nationalisme est développé à son paroxysme à la Révolution française. ${ }^{\text {xxi }}$ Autrement dit, le nationalisme est né bien avant les temps modernes (contrairement à Kedourie). Ceci pourrait expliquer pourquoi l'anti-nationalisme de Trudeau se base sur le fait que modernité et nationalisme sont inconciliables.

\section{L'évolution de sa pensée nationaliste}

a) L'anti-nationalisme 
La formulation de l'anti-nationalisme de Trudeau est nettement tributaire d'un contexte historique. Alors que le Québec des années 1940 et 1950 est traversé par un conservatisme fort, Trudeau rêve de transformer l'État québécois et canadien en État moderne où la liberté, l'égalité, la raison et le progrès domineraient. Autrement dit, le contexte historique l'amène à militer pour la modernité politique. Or, le nationalisme des années 1940 et 1950 est aussi tributaire de ce conservatisme : il est anti-moderniste, anti-émancipateur et ethnique. Devant ce dégoût de cette doctrine antimodernisatrice (qui ne changera pas avec les années), Trudeau conçoit une théorie politique moderne qui exclut tout nationalisme. ${ }^{\text {xxii }}$

On peut facilement mentionner que l'objectif politique de Trudeau est la réforme du système canadien pour en faire un État moderne libéral. Or, pour ce dernier, le nationalisme ne présente que des défauts pour tarmodernité. Il utilise cinq éléments pour prouver le tout. Premièrement, le progrès est un pilier très fort de la modernité politique. Trudeau donne deux éléments pour démontrer que le nationalisme n'est pas progressiste. Tout d'abord, Trudeau croit que le progrès passe par la compétition: "une culture ne progresse que par

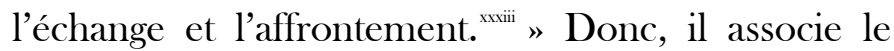
protectionnisme culturel à un retour en arrière : seul l'affrontement entre les cultures et les individus permet l'avancement des hommes. Ensuite, Trudeau développe un historicisme du progrès : «Or, l'histoire de la civilisation, c'est l'histoire de subordination du nationalisme "tribal" à des appartenances plus larges. ${ }^{\text {xxiv } " ~ A u t r e m e n t ~ d i t, ~}$
Trudeau croit que «le progrès de l'homme commande l'abandon de ces héritages qui nous divisent et la reconnaissance de la fraternité de tous dans la transparence du dialogue rationnel. Cet acte réfléchi de reconnaissance de soi dans l'autre, quel que soit son lieu dans l'histoire, constitue une voie vers un avenir meilleur. ${ }^{x x v}$ "On voit donc que le progrès passe par un universalisme rationnel, auquel l'histoire nous confère, qui déboucherait sur une paix et une fraternité mondiale. ${ }^{\text {xxxi }}$ Deuxièmement, comme Kedourie, Trudeau croit que la Raison, un pilier du modernisme, est incompatible avec la nation : « transi par l'héritage des Lumières, Trudeau considère que la maison de l'homme n'est pas la nation, mais bien la raison. ${ }^{\text {xxxii } " ~ A u t r e m e n t ~ d i t, ~}$ « l'homme n'appartient ni à sa langue, ni à sa race ; il n'appartient qu'à lui-même, car c'est un être libre, c'est-à-dire un être moral. ${ }^{\text {xxviii } " ~ D o n c, ~ p a r c e ~ q u e ~}$ l'individu se définit par son individualité et sa Raison, le nationalisme est non-nécessaire. En plus, en évacuant la Raison, il favorise la mise en place d'un régime démagogue, violent et sophiste. ${ }^{\text {xxix }}$ Finalement, la Raison donne de la dignité à l'humain : «pour [Trudeau], la dignité de l'homme lui vient de sa capacité de faire des choix éclairés. " " Ainsi, parce que le nationalisme véhicule l'affectivité, il évacue toute rationalité et dignité humaine. Troisièmement, pour utiliser sa Raison et avoir une dignité, l'individu ne doit avoir que des droits contre l'État (espace d'autonomie)..$^{\text {xi }}$ Ainsi, « la liberté ne se justifie pas par-elle même. ${ }^{\text {xli } ~ " ~ D o n c, ~ i l ~ d o i t ~ a v o i r ~ d e s ~}$ libertés fondamentales hors de l'atteinte de l'État. Comme Kedourie, Trudeau croit que le nationalisme absorbe les individus dans les 
institutions étatiques et que la liberté individuelle soit remplacée par celles collectives. Il craint donc que ces dernières libertés ne favorisent pas la Raison et la dignité humaine. Quatrièmement, la modernité affiche un souci important pour l'égalité (ou l'homogénéité). On comprend donc que, pour avoir une égalité homogène, Trudeau croit qu'il faut éliminer les différences et former un espace universel où tous les gens des mêmes communautés pourront avoir les mêmes droits et libertés et ainsi, la même dignité. On comprend donc la pertinence de la politique du multiculturalisme dans cette vision universelle. Cinquièmement et finalement, l'égalité homogène vise la tolérance. En dressant un État multiculturel, Trudeau fait en sorte que les différentes communautés sont égales et que ces dernières ne débouchent pas vers la persécution d'un groupe par un autre. Ainsi, tout nationalisme qui s'oppose à ceci et qui promeut des valeurs ethniques qui divisent débouche à l'intolérance : « un excès de protectionnisme culturel dégénère inévitablement en intolérance et, au bout du compte, en négation absolue de la liberté individuelle au nom de la dignité du groupe. ${ }^{\text {xiii } ~ " P o u r ~ r e ́ s u m e r, ~ o n ~ v o i t ~ d o n c ~ q u e ~ l a ~}$ modernité que Trudeau veut mettre sur pied est incompatible avec le nationalisme. Par contre, comme Kedourie, Trudeau identifie deux autres problèmes de nature plus pratique du nationalisme.

Comme Kedourie, Trudeau identifie deux problèmes politiques au nationalisme. Tout d'abord, il affirme que le nationalisme est responsable des pires crimes de l'histoire. ${ }^{\text {div }}$ Comme son maitre à penser, il associe nationalisme et violence mais de manière moins détaillée. Ensuite, tout comme
Kedourie, il associe le nationalisme à la déstabilisation. Dans un premier temps, il mentionne que toutes affirmations du nationalisme dans une fédération pluriethnique conduit à la destruction de la fédération. ${ }^{x v}$ Dans un second temps, il soutient que l'application du principe de nationalité apporterait la déstabilisation des relations internationales, comme le soutenait Kedourie. ${ }^{\text {xli }}$ On voit donc la proximité idéologique de ces deux hommes...

Ainsi, nous pouvons conclure que, durant cette période, Trudeau condamne le nationalisme ethnique à cause de son incompatibilité avec la modernité politique et la gestion politique. Un nationalisme civique politique est aussi condamnable, puisqu'il risque de développer l'affectivité et d'évacuer la Raison. ${ }^{\text {xlii }}$ Autrement dit, Trudeau confine tout le nationalisme «dans la sphère privée et l'inaliénabilité des droits individuels. xlviii "On voit donc que Trudeau et Kedourie sont sur la même longueur d'onde: association entre violence, déstabilisation et nationalisme et l'incompatibilité entre libéralisme moderne et nationalisme. Par contre, face au nationalisme québécois, Trudeau est forcé de changer son fusil d'épaule...

\section{b) Le nationalisme civique}

La tenue d'un référendum sur la souveraineté association et la montée du mouvement indépendantiste québécois sont un moment important dans le développement et l'évolution de la pensée de Pierre Trudeau. Ces événements démontrent la difficulté voire même l'échec de la concrétisation de sa pensée universaliste moderne. Par contre, le Premier ministre avait des munitions 
dans son sac: il "se résolut à recourir à l'épais ciment du nationalisme pour contrer ses adversaires de toujours, les souverainistes et les nationalistes du Québec. ${ }^{\text {xix }}$ "Il formule donc un nationalisme civique dont la Charte canadienne des droits et libertés sera le porte-étendard :

«[...] C'est le poids dans la balance du côté du provincialisme aux dépens d'une institution fédérale ou d'une législation, qui, jusqu'à présent, donnait aux Canadiens un sens d'appartenance nationale, un peu comme une Charte des droits et libertés était importante pour l'unité canadienne, un peu comme le rapatriement de la Constitution, un peu comme le drapeau canadien. Tout cela, c'est important en ce sens que ça fait comprendre aux Canadiens qu'ils partagent avec TOUS les Canadiens, de TOUT le pays, urr MÊME ensemble de valeurs fondamentales.

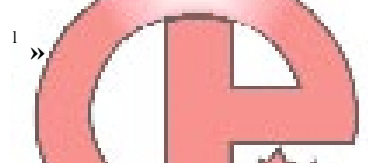

Autrement dit, étảnt donné que les circonstances actuelleš ne permettent pas lucidement aux individus d'abandonner leurs différences, Trudeau développe un nationalisme civique canadien puissant qui, à long terme, permettra d'en venir à une identité universelle. Pour le dire autrement, ce nationalisme, en rassemblant plusieurs communautés nationales sous le même emblème, est mis au service de la marche de l'histoire. Par contre, le nationalisme canadien de Trudeau respecte-t-il encore la modernité ? Nous allons maintenant nous attarder à cette problématique.

Dans le paragraphe précédent, nous avons vu que Trudeau n'a pas abandonné l'idée de faire du
Canada un État moderne (l'idée phare des antinationalistes). En effet, la volonté de concrétiser la marche de l'histoire vers l'universalisme, la Raison et la fraternité à travers le nationalisme civique témoigne de la situation. On voit donc que l'utilisation par Trudeau de l'affectivité a une utilité : parvenir à une plus grande Raison. Autrement dit, il demeure un Moderne, puisqu'il croit à la Raison. ${ }^{\text {li }}$ Parallèlement, dans la Charte, Trudeau met une série de libertés et de droits dans les mains des individus. Cette protection contre l'État laisse donc une zone «d'autonomie» pour l'utilisation de la Raison, confirmant ainsi le primat qu'il accorde à la rationalité. Ensuite, la nation canadienne est multiculturelle. Ceci témoigne d'un respect de trois principes libéraux modernes : l'égalité, la neutralité étatique et la tolérance. Finalement, le nationalisme de Trudeau croit à la concurrence des cultures à des fins progressistes. Pour le dire autrement, la Charte favorise la compétition entre les groupes afin qu'il progresse et abandonne leurs particularismes. ${ }^{\text {lii }}$ D'ailleurs, Trudeau a longuement affirmé que le Canada ne devait pas donner des droits particuliers aux autochtones; ces derniers doivent devenir membre à part entière de la communauté canadienne « universelle ». liii On voit donc que, par la concurrence des communautés dans la même nation, le nationalisme civique canadien vise le progrès vers la communauté de plus en plus universelle, comme le veut l'historicisme libéral de Trudeau et les thèse antinationalistes. Pour conclure, ce penseur théorise un système très près de la modernité avec un nationalisme qui, malgré le désavantage d'affectivité qu'il possède, a l'avantage de conduire vers la 
communauté fraternelle et universelle. En ce sens, on note donc l'influence marquée de l'antinationalisme sur ses idées politiques.

\section{Conclusion}

En somme, Kedourie et Trudeau sont des auteurs très importants pour la compréhension du phénomène national et par le fait même, du fédéralisme. Bien que ces auteurs ne fassent pas l'unanimité, ces auteurs rappellent la dérive facile du nationalisme vers la violence et la déstabilisation. Par contre, le grand mérite de ces intellectuels est de démontrer d'une façon claire et lucide les tensions intrinsèques que le nationalisme entretient avec la modernité. Il faut cependant noter que, depuis les années 1990, d'autres intellectuels (notamment Will Kymlicka et Charles Taylor) s'acharnent à prouver l'inverse, à savoir que la modernité ne doit pas évincer la nation. D'ailleurs, nous sommes forcés de constater que l'anti-nationalisme de Trudeau et Kedourie semble mal résister à la réalité historique. Devant l'affirmation d'un sentiment communautaire très fort tet le désir d'obtenir des droits communautariens chez les certains immigrants canadiens, l'antinationalisme ou le nationalisme civique de Trudeau (grandement inspiré par Kedourie) semble avoir créé une résurgence des mouvements identitaires alors que l'affirmation de la modernité libérale dans la politique du multiculturalisme désirait clairement estomper les différences. liv Peut-on conclure à l'échec de la politique multiculturaliste moderniste ou au fait qu'elle va trop ou pas assez loin? Des études supplémentaires devraient être réalisées pour avoir une conclusion hors de tout doute.
${ }^{\mathrm{i}}$ KYMLICKA Will, La voie canadienne : repenser le multiculturalisme, Montréal, Boréal, 2003, p.170-189

${ }^{i i}$ Voir le compte-rendu de JACQUES Daniel, Humanités passagères, Montréal, Boréal, 1991, p.49-50

iii LAFOREST Guy, « Herder, Kedourie et les errements de l'anti-nationalisme », in De la prudence, Québec, Boréal, 1993, p.59 et $\mathrm{p} .61$

iv KEDOURIE Elie, Nationalism, London, Hutchinson University Library, 1966, p.72

${ }^{\vee}$ LAFOREST Guy, op. cit., p.61

${ }^{v i}$ SMITH.D Anthony, Nationalism, London, Ed. Polity, 2001, p. 48

vii Voir notamment GELLNER Ernest, Nationalism, New York University Press, 1997 et Benedict ANDERSON,

L'imaginaire nationale : réflexions sur l'origine et l'essor du nationalisme, Paris, La Découverte, 2002.

viii LAFOREST Guy, op. cit., p.61-62

${ }^{\text {ix }}$ SMITH Anthony D., « The myth of the Modern Nation and the myths of nations » in Ethnic and Racial Studies, vol.11, no.1, p.3-4

${ }^{x}$ MACLURE Jocelyn, Récits identitaires, Québec, Québec

Amérique, 2000, p.134

${ }^{\mathrm{xi}}$ KEDOURIE Elie, op. cit., 92-93

xii Ibid., p.111

${ }^{\text {xiii }}$ LAFOREST Guy, op. cit., p.59

${ }^{\text {xiv }}$ KEDOURIE Elie, op. cit., p. 130

${ }^{\mathrm{xv}}$ Idem, p. 109.

${ }^{\mathrm{xvi}}$ LAFOREST Guy, op. cit., p.62-63

xvii Idem.

xviii KEDOURIE Elie, op. cit., p.117

${ }^{\text {xix }}$ LAFOREST Guy, op. cit.,, p.63-65 et Elie KEDOURIE, op cit., p.117

${ }^{\mathrm{xx}}$ KEDOURIE Elie, op. cit., p.29

${ }^{\mathrm{xxi}}$ LAFOREST Guy, op. cit.,, p.63-65

${ }^{x x i i}$ KEDOURIE Elie, op. cit., p.31

xxiii Idem., p.48

xxiv Idem., p.48-87-88

${ }^{\mathrm{xxv}}$ Idem., p.56

${ }^{\mathrm{xxvi}}$ Idem., p.89

xxvii Ibid., p.45

xxviii MACLURE Jocelyn, op. cit., p.138

xxix KELLY Stéphane, Les fins du Canada, Québec, Boréal, 2001,.p.204

${ }^{\mathrm{xxx}}$ TRUDEAU Pierre Elliott, « La nouvelle trahison des clercs » in Le fédéralisme et la société canadienne-française, Montréal, Éd. HMH, 1967, p..166

xxxi Idem.

xxxii KELLY Stéphane, op. cit., p.205

xxxiii BICKERTON James , GAGNON Alain G. et BROOKS

Stephen, Six penseurs en quête de liberté, d'égalité et de communauté, Québec, Presses de l'Université Laval, 2003, p. 140

xxxiv TRUDEAU, Pierre Elliott, op. cit., p.165

${ }^{\mathrm{xxxv}}$ JACQUES Daniel, op. cit., p.49-50

xxxvi Idem.

xxxvii MACLURE Jocelyn, op. cit.,p.140

xxxviii TRUDEAU Pierre Elliott, «Fédéralisme, nationalisme et raison » in Le fédéralisme et la société canadienne-française, op. cit., p. 207 
xxxix Idem.

${ }^{\mathrm{xl}}$ BICKERTON James, GAGNON Alain G. et BROOKS

Stephen, op. cit., p.133

xli Idem.

xlii Idem.

xliii Idem., p.139

xliv TRUDEAU Pierre Elliott, « La nouvelle trahison des clercs », op. cit., p.170-176

xlv TRUDEAU Pierre Elliott, « Fédéralisme, nationalisme et raison » in Le fédéralisme et la société canadienne-française, op. cit., p.207

xlvi TRUDEAU Pierre Elliott, « La nouvelle trahison des clercs », op. cit., p.161

xlvii TRUDEAU Pierre Elliott, « Fédéralisme, nationalisme et raison " in Le fédéralisme et la société canadienne-française, op. cit., p.204 à 208

xlviii MACLURE Jocelyn, op. cit., p.149

${ }^{x}$ LAF LAREST Guy, Pour la liberté d'une société distincte : parcours d'un intellectuel engagé, Québec, Presses de l'Université Laval, 2004, p.9

${ }^{1}$ TRUDEAU Pierre Elliott, « Il doit y avoir un sens d'appartenance » in JOHNSTON, Daniel (textes réunis et présentés par), Lac Meech. Trudeau Parle, Québec, Ed. Hurtubise HMH, 1989, p.41

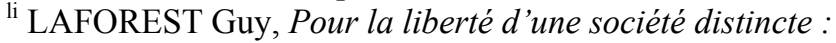
parcours d'un intellectuel engagé, op. cit.,p.11 à 19

lii KELLY Stéphane, op.cit., p.201

liii Idem.

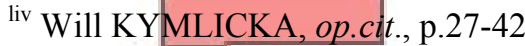

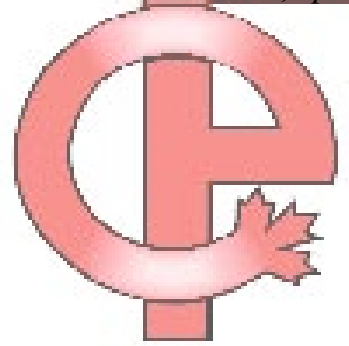




\section{Bibliographie}

ANDERSON, Benedict, L'imaginaire nationale : réflexions sur l'origine et l'essor du nationalisme, Paris, La Découverte, 2002, 212p.

BICKERTON, James, Alain G. GAGNON et Stephen BROOKS, Six penseurs en quête de liberté, d'égalité et de communauté, Québec, Presses de l'Université Laval, 2003, 187p.

GELLNER, Ernest, Nationalism, New York University Press, 1997, 114p.

JACQUES, Daniel, Humanités passagères, Montréal, Boréal, 1991, 288p.

JOHNSTON, Daniel (textes réunis et présentés par), Lac Meech. Trudeau Parle, Québec, Ed. Hurtubise HMH, 1989, 167p.

KEDOURIE, Elie, Nationalism, London, Hutchinson University Library, 1966, 140p.

KELLY, Stéphane, Les fins du Canada, Québec, Boréal, 2001, 283p.

KYMLICKA, Will, La voie canadienne : repenser le multiculturalisme, Montréal, Boréal, 2003, 339p.

LAFOREST, Guy, De la prudence, Québec, Boréal, 1993, 234p.

LAFOREST, Guy, Pour la liberté d'une société distincte : parcours d'un intellectuel engagé, Québec, Presses de l'Université Laval, 2004, 353p.

MACLURE, Jocelyn, Récits identitaires, Québec, Québec Amérique, 2000, 219p.

SMITH,Anthony

SMITH, Anthony D., « The myth of the Modern Nation and the myths of nations » in Ethnic and Racial Studies, vol.11, no.1, p.1-19

TRUDEAU, Pierre Elliott, Le fédéralisme et la société canadienne-française, Montréal, Éd. HMH, 1967, $227 p$. 